\title{
The effect of vertical tongue loading on the position perception of the tongue
}

\author{
MAN-TAK LEUNG and VALTER CIOCCA \\ University of Hong Kong, Hong Kong, China
}

\begin{abstract}
We investigated the effects of vertical tongue loading on the position perception of the tongue. Five male and 5 female university students served as subjects. Vertical upward and downward loading forces were applied to the tongue of the subjects. Their task was to judge the perceived horizontal position of the tongue after tongue-loading directions. The means of the judgments for the control conditions (no tongue loading) were compared with the judgments for perceived horizontal position after tongue loading. The results showed that vertical tongue loading produced a shift in the perceived horizontal direction opposite to the applied force. These results fully replicated the analogous aftereffect found by Grover and Craske (1991) for horizontal tongue loading. However, the judgments of perceived horizontal position in the present study had lower variability than did those in Grover and Craske's study, suggesting that mapping of the tongue along the vertical axis is more precise than mapping along the horizontal axis.
\end{abstract}

The tongue is an organ with a "skeleton" comprising muscles and connective tissues. Instead of attaching to a bony structure like the muscular structures in other parts of the body, the intrinsic muscles of the tongue are attached to the fibrous midline septum and the deep connective tissue of the lingual mucosa (Zemlin, 1981). Whereas limb movements are produced by the contraction of the skeletal muscle, which acts on a joint that lies between the origin and insertion of the muscle, the tongue is a joint-free hydrostatic structure that has no steady shape (Grover \& Craske, 1991; Zemlin, 1981). These structural characteristics imply that a unique mechanism of movement control may be involved for the tongue; in fact, the tongue is a very flexible articulator, capable of achieving numerous postures (Fucci \& Lass, 1999; Hayden \& Square, 1994). During speech production, the tongue movements and configurations play a major role in contrasting different phonemes by setting the place and degree of constriction along the vocal tract. The complexity of these configurations poses great difficulties in measuring and designing systematic perturbation. Research on tongue perturbation aims at improving our understanding of how

This article is based on a dissertation submitted by M.-T.L. in partial fulfillment of the requirements for a PhD degree at the University of Hong Kong, June 2001. We thank our subjects, who were willing to go through hours of unpleasant tongue-loading procedures. We are grateful to our technician, Donald Chan, who built the headset of the experiment, and we also thank W. Hardcastle, B. Murdoch, T. Whitehill, J. Kingston, and one anonymous reviewer, who gave valuable comments and detailed feedback on this study. Correspondence concerning this article should be addressed to should be addressed to M.-T. Leung, Department of Speech \& Hearing Sciences, University of Hong Kong, 5/F, Prince Philip Dental Hospital, 34 Hospital Road, Sai Ying Pun, Hong Kong, China (e-mail: mtleung@hkusua.hku.hk).

Note-This article was accepted by the previous editorial team, headed by Neil Macmillan. this unique hydrostatic structure contributes to the production of speech. The introduction of external load or perturbation is a powerful experimental method of capturing the dynamic moment-to-moment control properties of the neuromotor systems (Barlow, 1998). The application of load to limb (Evarts, 1981) and the application of load to the orofacial systems (Abbs \& Gracco, 1984; Gracco \& Abbs, 1985, 1988; Kelso, Tuller, VatikiotisBateson, \& Fowler, 1984) have been used to investigate the adaptive mechanism of human movements to the sudden application of external load.

It is well known that position sense is important for any organ operating in a spatial context (Broekhuijsen \& van Willigen, 1983; McCloskey, 1978). In the absence of the ability to sense the position of a limb, one would lose the control of the limb and would not be able to direct it to perform purposeful motions. As far as speech production is concerned, the tongue is an organ operating in space for the purpose of producing intelligible speech. The existence of a position sense should be even more critical for the tongue than for the limbs, because no visual information is available to substitute for the kinesthetic feedback to direct the tongue during speech production. The idea that our capacity to speak properly relies on our ability to sense the spatial position of the tongue is supported by studies on speech motor control (MacNeilage, 1970; Perkell, 1981).

For orofacial movements, vision is not a dominant factor for ongoing motor control. Therefore, the nervous system must rely on auditory, tactile, and proprioceptive information. The auditory feedback system makes use of the mechanoreceptors situated in the inner ear, but since it involves transmission of sound through the air or the bones, some investigators have found evidence that feedback through the auditory channel is slower than through the tactile and the proprioceptive channels. For example, Gracco (1995) reported that the average latency of the re- 
sponse to auditory information is on the order of $150 \mathrm{msec}$, which is too long to play an important role in the momentto-moment on-line monitoring of speech movements. Most sensory fibers of the tactile receptors in the oral region are contained in the trigeminal, vagus, and glossopharyngeal cranial nerves, and project in the somesthetic cortex in specific areas that correspond to areas of the body (Zemlin, 1981). The tactile receptor systems enable information to be sent to the central nervous system concerning the localizations of contacts. The receptor organs contributing to proprioceptive feedback are the muscle spindles, the joint receptors, and the Golgi tendon organs (GTOs), which serve to protect the corresponding muscles from exerting too much tension. Since the tongue is a jointless structure, the spindle is considered the major feedback information provider for the present study (Hardcastle, 1976). Muscle spindles are connected to fibers of high conduction velocity. It is well known that muscle spindles transmit information much faster than that provided by the tactile and auditory system, and that spindles provide information about the velocity and the amount of muscle stretch and tension of the corresponding muscles (Hardcastle, 1976). Given these properties, muscle spindles are likely to play an important role in orofacial motor control by setting a muscle reflexively to attain a specific length at any given time according to the target configuration (Brooks, 1986; MacNeilage, 1970). The target configuration can thus be set without continuous motor commands being sent from a higher center. Given its rapidity, proprioceptive information is likely to be available during speech production (Borden, Harris, \& Raphael, 1994). Moreover, the information about the rate of change of muscle length would enable the central nervous system (CNS) to compute the probable length of a muscle after a given period of time, thus providing the CNS with predictive information (Borden et al., 1994).

Since the tongue cannot rely on the information provided by the joint sensors and the bony skeleton, the feedback mechanism of the tongue must be different from that of the limbs and the jaw. The tongue may make use of afferent information from the muscle spindles during and after muscle contraction in response to the corollary discharge from the brain associated with voluntary movement (Lowe, 1981). Present anatomical understanding of muscle spindles is that they lie parallel to the main muscle fibers, and that the central parts of these spindles contain receptors that are sensitive to the changes in muscle length (Darley, Aronson, \& Brown, 1975). Since muscle spindles lie parallel to the main muscle and share the same attachments of the muscle to the structures in question, they are subjected to tension when the muscle is stretched and are relaxed when the muscle is shortened. When the muscle spindle of a main muscle fiber is stretched, the receptors in the muscle spindle fire, causing the main muscle fiber to contract and shorten its length until the muscle spindle is relaxed.

Grover and Craske (1991) investigated whether the tongue has a position sense and whether the lingual mu- cosa of the tongue may contribute important information about tongue position. They studied the performance of subjects with and without anesthesia of the lingual mucosa (4\% Xylocaine was administered to the upper and lower surfaces of each subject's tongue) after a 29.5 -g weight was applied to the tongue. A headset was used to secure each subject's head position during the experiment by means of a nose bar, a chinrest, and a head strap. At the beginning of the experimental (tongue-loading) trials, the tip of the subject's tongue was inserted into a plastic tongue holder, which was attached by a light nylon thread to a pulley system. The weight was attached at the other end of the pulley system. When the weight was released, it generated a force that pulled the subject's tongue to the left or to the right along the horizontal plane. The subjects were asked to hold the tongue straight ahead against the force of the weight for $1 \mathrm{~min}$. At the end of the 1-min period, the weight attached to the tongue was freed from the tongue holder, and the subjects were asked to judge the straight-ahead position along the horizontal plane by protruding the tongue. During judgment, the tongue rested on a horizontal plastic sheet that was fixed on the headset and was adjusted at the lower lip level of each subject. The judgments of the straight-ahead position made before and after tongue loading were compared. It was found that, for most subjects, there was an aftereffect produced by tongue loading: The aftereffect caused the perceived position of the tongue to be shifted along the horizontal plane toward the direction of the previous effort when compared with the judgments made before tongue loading. For example, if the weight had pulled the tongue to the left at the beginning of a trial, the perceived straight-ahead position along the horizontal plane was judged to be to the right of the judgments made without tongue loading. Grover and Craske concluded that the tongue operated in the same manner as the jointed bony segments to which most skeletal muscles are attached. Since they found no effect of the anesthesia, they argued that cutaneous sensation does not contribute to the position sense, whereas the spindles in the tongue were likely to contribute to the observed aftereffect. Hutton, Smith, \& Eldred (1973) and Lowe (1981) demonstrated that a muscle that has just borne a load continues to send out afferent information (postcontraction discharge) after the removal of the load. The observation that effortful muscular work biased the position sense of the tongue as observed was viewed by Grover and Craske to be due to a postcontraction effect. They suggested that, after tongue loading, the spindles in the tongue continue to supply sensory information, which contributes to the wrong interpretation of the position of the tongue along the horizontal axis after tongue loading.

The demonstration that subjects can perceive the position of the tongue along the horizontal plane is important because it provides the basis for investigating the perceived position of the tongue along other planes. Relative to the existence of position sense along the horizontal plane, the existence and characteristics of position sense in the vertical plane have a more direct signifi- 
cance to speech production. In terms of speech production, lateral tongue movements along the horizontal plane are not part of the phonological systems of most languages (Ladefoged \& Maddieson, 1996). For most languages, the tongue movements that are most often used to convey linguistic information are those that occur along the vertical plane and along the forwardbackward plane (Ladefoged, 1975). In addition to movements on the vertical and forward-backward planes, the "lateral consonants" are pronounced by the contraction of the transverse muscles of the tongue, producing an occlusion along the midsaggital plane of the vocal tract but with airflow around the occlusion, whereas the retroflex sounds are produced with curling of the tongue tip. The perceived position of the tongue on the vertical plane is necessary to produce phonetic distinctions among English front vowels (for example, the distinction between /i/ and / $\varepsilon$ / in English), for which the tongue movements along the other planes are relatively minimal (Ladefoged, 1975). Therefore, the aftereffect following vertical tongue loading could be a useful tool for understanding the relationship between tongue position sense and speech production.

The main goal of the present study was to investigate the shift in the perception of tongue height induced by vertical tongue loading. To do this, the loading forces were applied vertically (that is, upward and downward relative to the straight-ahead position) rather than laterally. If tongue loading biases the perceived tongue position toward the direction of the previous action, the judged perceived horizontal position of the tongue should be different from that of the control condition (no tongue loading). For example, if an upward vertical pull is applied, the subject will have to resist the pressure by pushing his/her tongue downward in order to keep the tongue in the perceived horizontal position. Therefore, his/her perceived position of the tongue after an upward pull should shift downward, which should cause judgments of the perceived horizontal position to be lower after an upward pull than in the control condition. The opposite pattern should be observed following a downward pull of the tongue before judgments of the perceived horizontal position are made.

\section{METHOD}

\section{Subjects}

Thirteen speech therapy students at the University of Hong Kong participated in the study ( 7 male and 6 female; mean age $=21$ years). They all had normal pure tone hearing thresholds (within $15 \mathrm{~dB}$ $\mathrm{HL}$ ), and no articulation problem as judged by the first author, who is a certified speech therapist. The subjects were naive about the aim of the experiment. The results of 3 of the 13 subjects were not entered in the data analysis because of the following reasons. Two female subjects found the suction generated by the tongue holder unbearable, and gave up after finishing the first block of experimental trials; one male subject could not follow the instructions to move his tongue in the manner required for making the judgments of the perceived horizontal position along the vertical axis. Consequently, only the data of 5 male and 5 female subjects were analyzed.

\section{Materials}

A headset $(25 \mathrm{~cm}$ deep $\times 32 \mathrm{~cm}$ wide $\times 45 \mathrm{~cm}$ high $)$ was used to keep the subject's head in position during the experiment (see Figure 1). It was made of transparent acrylic plastic, and it incorporated a padded nose bar (A), a chinrest (B), a pair of adjustable cheek pads (C) and a head strap (D). The front part of the headrest

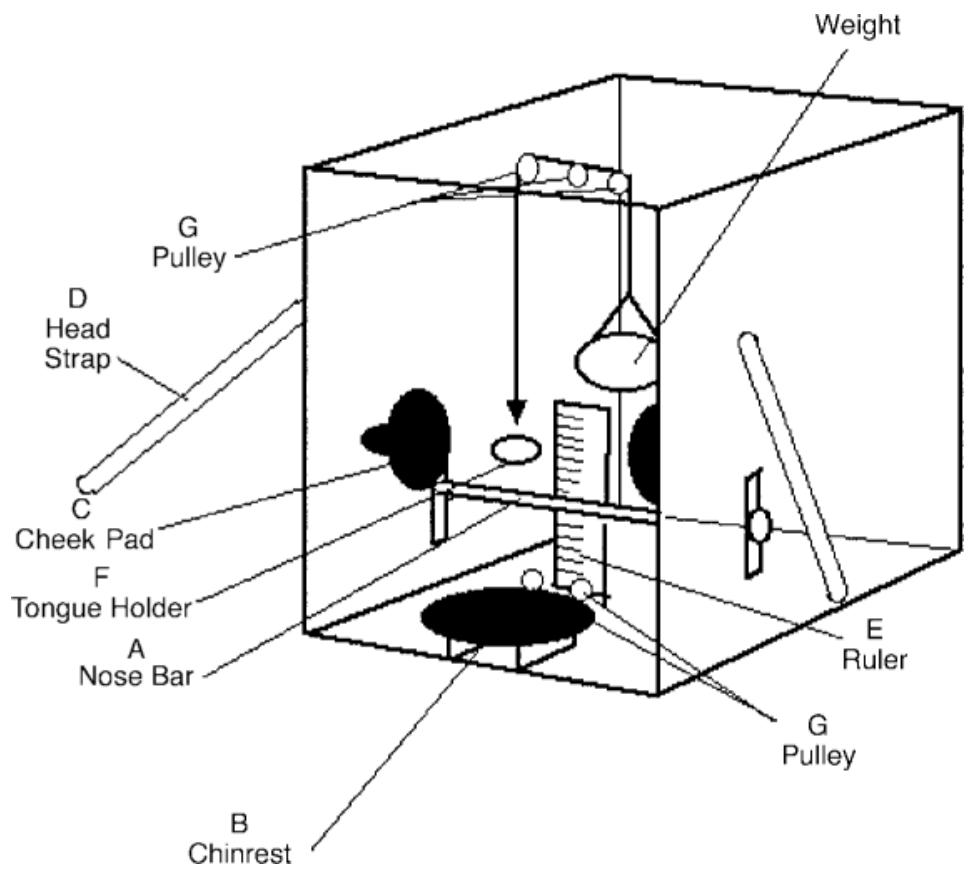

Figure 1. Diagram of the headset used for tongue loading. 
supported a detachable ruler (E) with millimeter marks. The ruler was trimmed so that the lowest point where the ruler was attached to the base of the headset indicated $0 \mathrm{~mm}$. This ruler was used to measure the perceptual judgments of horizontal tongue position, as the distance on the vertical axis between the position of the tongue and the position of the upper edge of the lower incisors, which was different for each subject. In Grover and Craske's (1991) experiment, a weight of $29.5 \mathrm{~g}$ was used. Therefore, we used a weight of $30 \mathrm{~g}$ to generate the loading force in the present experiment. The weight was attached with a piece of lightweight, 22-cm-long nylon thread to a plastic tongue holder $(F)$. The thread passed through one of two pulley systems $(\mathrm{G})$; one pulley system was used for exerting an upward-loading force and the other for a downward-loading force (see Figure 2). A video camera (JVC Model GR-AX7E) was positioned directly in front of the headset at a distance of $50 \mathrm{~cm}$. The focus of the camera was fixed on a detachable ruler, inserted in the headset in order to record the perceptual judgments of horizontal tongue position and the position of the upper edge of the subjects' lower incisors.

\section{Procedure}

Following Grover and Craske's (1991) procedure, there were two control conditions, "basic control" and "tongue protrusion," and one experimental condition, "tongue loading." Each condition was run in a separate block of trials. In all trials, the subjects were required to judge horizontal position of the tongue by protruding the tongue, and by pointing it straight ahead along the vertical axis. In the basic control condition, the subjects simply made 10 judgments at 5 -sec intervals. In the tongue-loading condition, the subjects made 10 judgments after a downward or upward pull had been applied to the tongue for a period of $30 \mathrm{sec}$. In the tongue protrusion condition, 10 judgments were made after the subjects had protruded the tongue in the perceived horizontal position for an uninterrupted period of $30 \mathrm{sec}$. The tongue protrusion condition was included to ensure that any aftereffects produced by tongue loading were due to the vertical pull of the weight, and not to the exertion of effort required to protrude the tongue straight ahead. If tongue protrusion per se did not produce significant shifts in the perceived position of the tongue, the basic control and the tongue protrusion conditions would not be expected to produce statistically significant different judgments of the perceived horizontal position. Our main prediction for this experiment was that vertical tongue loading would shift the perceived position of the tongue by producing an aftereffect similar to that reported by Grover and Craske. If this was the case, judgments of the perceived horizontal position in the tongue-loading conditions should be significantly higher (for downward loading) or lower (for upward loading) than those in both the basic control and the tongue protrusion conditions.

This experiment was composed of two sessions. Within each session, and before the actual data collection, each subject was asked to make 10 judgments after a 30 -sec tongue-loading period, to become familiar with the procedure. Two min after the familiarization, the basic control, the tongue protrusion, and the tongue-loading conditions followed each other in this order. During the tongue-loading conditions, in each block of trials, the subject's tongue was pulled either upward (upward tongue loading), or downward (downward tongue loading). The order of upward and downward tongue-loading conditions was balanced such that half of the subjects had the downward loading condition during the first session and the upward loading condition during the second session; for the remaining subjects, the order of the upward and downward loading sessions was reversed. The subjects had a rest period of at least 30 min between the two sessions. The two sessions were run within a single day, requiring about $2 \mathrm{~h}$ to complete (not including the rest period).

At the beginning of a block of trials, the subject was instructed to place his/her head in the headset. Once the headrest and the rest

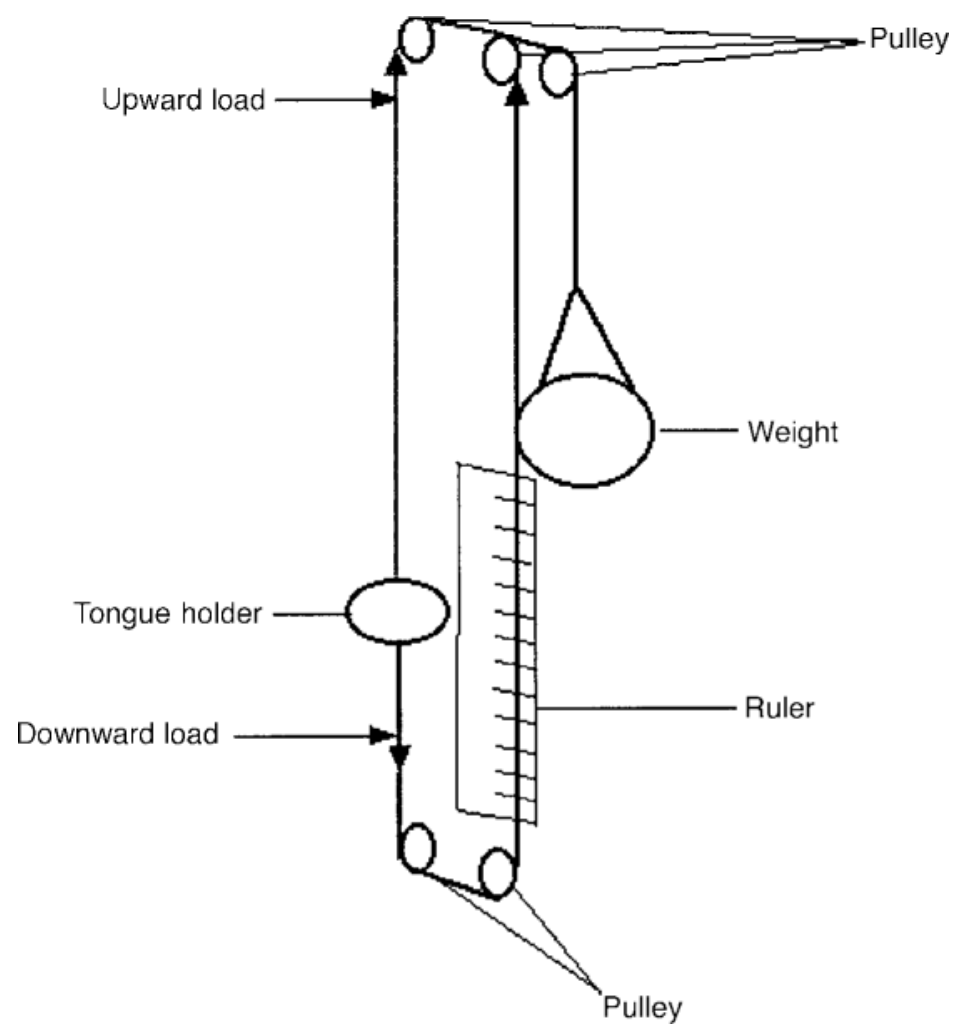

Figure 2. The pulley system for generating the upward and downward forces. 
of the apparatus had been fitted, the experimenter fastened the head strap around the subject's head and started the videotape recording. The subjects were instructed to shut their eyes at this point, and to keep them shut throughout each block (i.e., until the last judgment of the perceived horizontal position of the tongue in each block had been completed) in order to avoid using visual cues for making the judgments. Then, the subject was asked to open his/her mouth so that the lower jaw was stationed on the chinrest, and the nose rested on the nose bar. When the subject felt comfortable with the head position, the experiment began. In the "basic control" condition, the subjects were asked to make tongue position judgments once the headrest and the accompanying apparatus had been adjusted. In the tongue protrusion condition, the subjects were asked to protrude the tongue for $30 \mathrm{sec}$. The ruler was detached from the headrest in this phase of the experiment to avoid the subject's touching the ruler with the tongue. When the $30 \mathrm{sec}$ were over, the ruler was placed back in position while the subject retracted the tongue. The subject was instructed to retract the tongue between successive trials (i.e., after each judgment). Before the beginning of the tongue-loading conditions, the subject sucked a plastic tongue holder of negligible weight onto the tongue tip and positioned the head in the headset. Then, the subject protruded the tongue, and the weight was gently released over the pulley. The subject was instructed to maintain the tongue straight ahead for $30 \mathrm{sec}$ against the force applied. Once $30 \mathrm{sec}$ had elapsed, the experimenter released the suction and removed the tongue holder from the subject's tongue.

The experimenter instructed the subject to judge when the tongue was felt to be horizontal using the method of adjustment. When the experimenter called out "from above," the subject first protruded the tongue and pointed it upward; then the subject moved the tongue along the ruler until the tongue was felt to be straight ahead along the vertical axis (i.e., "parallel to the floor"). The subjects were advised to adjust the tongue position slowly and were allowed to backtrack if they thought they had gone too far. When a subject was satisfied with the placement of the tongue, he/she knocked on the table to indicate that the judgment had been completed. Then the subject retracted the tongue and waited for the next trial to begin. Each judgment took about $3 \mathrm{sec}$ to complete. When the experimenter called out "from below," the same procedure was repeated except that the subject started a judgment by pointing the tongue down- ward. Trials starting from "above" or "below" alternated for each subject, for a total of 10 successive trials at 5-sec intervals for each condition. After completing a session, the subject exited the headrest and rested for at least $30 \mathrm{~min}$.

To summarize, each subject made a total of 60 judgments of the perceived horizontal position along the vertical axis: two sessions (upward and downward loading) $\times$ three conditions (basic control, tongue protrusion, and tongue loading) $\times 10$ judgments.

\section{Measurements and Analysis}

The videotaped sessions were played back on a television set so that the experimenter could check that the subjects followed the instructions to keep their eyes closed during each judgment. All judgments of the 10 subjects were entered in the analysis. For each judgment, the videotape was paused at the point when the subject's knock was heard. Measurements were made from the paused image on the television set. The point where the tongue was making contact with the ruler, which usually appeared to be somewhat like a circle dot (see Figure 3), was used for the measurements. The "top margin" of the tongue was estimated by obtaining a reading in millimeters from the ruler of the uppermost point of contact of the tongue with the ruler; the "bottom margin" was measured as the lowest point of contact as indicated in Figure 3. The millimeter ticks that were closest to the upper and lower marks of the contact of the tongue with the ruler were taken as the "top" and "bottom" margin measurements, respectively. The tape was then rewound to the point where the subjects started to protrude their tongues for that particular judgment, so that the height of the upper edge of the lower incisors above the base of the headset could be recorded. Although the chin and the ruler were in a fixed position within the same block of trials, the position of the subjects' upper edge of lower incisors varied slightly among blocks because of small head movements. To account for these changes in the placement of the head within the headset, the level of the upper edge of the lower incisors was taken as an indicator of the lower jaw position and acted as the reference point for the measurement of the perceived horizontal position. The measurement of the judged perceived horizontal position of the tongue was then calculated as the distance of the midpoint between the top and bottom margins from the upper edge of the lower incisors. The following formula was used to calculate tongue place-

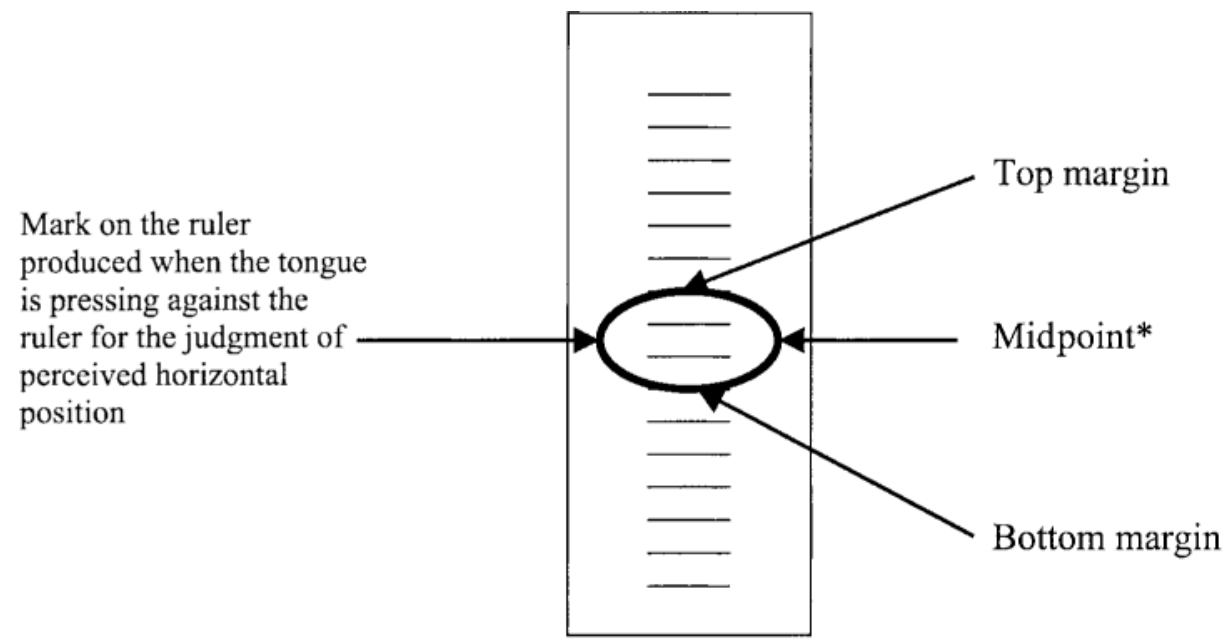

$*$ Midpoint $=$ bottom margin $+[($ top margin - bottom margin $) / 2]$

Figure 3. Measurement of the perceived horizontal position. 
ment within a trial: Straight-ahead position $=$ bottom margin + [(top margin - bottom margin)/2] - upper edge of lower incisors. The "top margin," "bottom margin," and "upper edge of lower incisors" were the readings (in millimeters) from the ruler.

Since the camera was always placed directly in front of the subject's mouth, errors in the measurement of the midpoint of the tongue and of the upper edge of the lower incisors from the videotape were expected to be rather small and consistent across subjects and conditions. A reliability check on data measurements was conducted, using an observer naive with respect to the purpose of the experiment. The naive observer calculated measurements of the judgments of the position of the tongue for a total of 30 trials: 10 randomly selected tongue protrusions, 10 basic controls, and 10 tongue-loading conditions using the procedure mentioned above. The correlation between the measurements calculated by the two observers was high (Pearson's $r=.94$ ).

\section{RESULTS}

The means of the 10 judgments for each condition and each subject were considered as the estimates of the perceived straight-ahead position. The means for each subject, as well as the overall means for each condition, are shown in Table 1. This table shows that, after downward loading, all subjects shifted the perceived horizontal position in the expected direction (upward), except Subject 8, whose perceived horizontal judgment was higher (downward) than that for the basic control but lower than the protrusion control (upward). After upward loading, all subjects, with the exception of Subjects 6, 8, and 9, shifted the perceived tongue position downward, as expected. Since Subjects 6, 8, and 9 were males, it is possible that the weight used for loading might not have been heavy enough for them to produce a consistent loading effect.

Since the tongue protrusion condition was included to control for perceptual shifts in the tongue position due to tongue protrusion per se, a preliminary two-way repeated measures analysis of variance (ANOVA) was carried out to determine whether the basic control and the tongue protrusion conditions produced significantly different judgments. The factors were the control condition (basic control vs. tongue protrusion) and the sessions (upward

Table 1

Mean Judgments (in Centimeters) of the Perceived Horizontal Position From the Ruler Attached to the Headset

\begin{tabular}{cccrrrrr}
\hline & \multicolumn{3}{c}{ Upward Loading } & & \multicolumn{3}{c}{ Downward Loading } \\
\cline { 2 - 3 } \cline { 6 - 7 } Subject & Basic & Protrusion & & & Basic & Protrusion \\
& Control & Control & Loading & Control & Control & Loading \\
\hline 1 & 1.04 & 0.83 & 0.65 & & 1.05 & 1.08 & 1.84 \\
2 & 0.20 & 0.14 & -0.01 & -0.05 & -0.45 & 0.05 \\
3 & 0.68 & 1.02 & 0.41 & & 0.63 & 0.48 & 1.13 \\
4 & 1.31 & 1.47 & 0.79 & & 1.15 & 1.13 & 1.40 \\
5 & 1.23 & 1.15 & 0.24 & & 0.96 & 1.05 & 1.12 \\
6 & 0.34 & 0.06 & 0.52 & & 0.41 & 0.43 & 0.86 \\
7 & 0.59 & 0.51 & 0.30 & -0.06 & 0.02 & 0.39 \\
8 & 1.27 & 0.85 & 1.27 & & 0.70 & 1.18 & 0.94 \\
9 & 0.00 & -0.20 & 0.01 & & 0.00 & 0.03 & 0.25 \\
10 & 0.77 & 0.58 & 0.12 & & 0.31 & 0.38 & 0.65 \\
$M$ & 0.74 & 0.64 & 0.43 & & 0.51 & 0.53 & 0.86 \\
$S D$ & 0.44 & 0.50 & 0.38 & 0.44 & 0.53 & 0.52 \\
\hline
\end{tabular}

vs. downward loading). Neither the main or interaction effects, nor the differences between pairs of conditions were found to be statistically significant. Following this result, the averages of the 10 judgments for the basic control and the tongue protrusion conditions within each experimental block were pooled together. A second twoway repeated measures ANOVA was carried out to compare the means of the judgments for the pooled control conditions with the judgments of the tongue position after loading. The two factors were the "loading condition" (control vs. loading) and the "loading direction" (upward vs. downward). The results of the ANOVA showed that there was a significant "loading condition" $\times$ "loading direction" interaction effect $[F(1,9)=43.7, p<.0001]$. Figure 4 shows that after an upward tongue loading, the mean perceived horizontal position was lower (i.e., shifted downward) than the judgment made in the control condition; this difference was statistically significant (Tukey HSD, $p<.05)$. The downward loading of the tongue significantly shifted the perceived position of the tongue judgment upward, relative to the judgments produced in the control condition (Tukey HSD, $p<.001$ ). The difference between the means of the control trials under the two loading conditions was not statistically significant (Tukey HSD, $p>.05$ ). None of the main effects was statistically significant. Since the basic control and the tongue protrusion conditions in the two loading direction blocks did not differ significantly from one another, the shift in the judgment of the straight tongue position along the vertical axis can be attributed to the aftereffect of tongue loading. Therefore, the results of this experiment showed that a vertical tongue loading produced a shift in tongue position perception in the opposite direction of the applied force. These results fully replicated the analogous aftereffect found by Grover and Craske (1991) for horizontal tongue loading.

In order to investigate the time course of the aftereffect, the means of the judgments for each subject after downward and upward loading were plotted as a function of the serial position of trials within each block (Figure 5). A linear regression was carried out to inspect the linear trend over time for judgments made in the two loading directions. No significant linear trend was found for the downward loading data. However, a significant linear trend was found for the upward loading data $(r=$ $.84, p<.005$ ), indicating that the effect of tongue loading was decreasing over time for this loading condition. The regression line for the upward loading data has the following equation: $y$ (time interval) $=a+b x$ (perceived horizontal judgment), where $a=-0.423 ; b=$ 0.028 . On the basis of this equation, and considering that each judgment occurred at every $3 \mathrm{sec}$, the aftereffect of tongue loading would effectively vanish at $45.3 \mathrm{sec}$ after the beginning of the trials. These findings are different from those of Grover and Craske (1991), who found no significant linear trend for the left and right loading data and commented that the high variability of their data contributed to the failure to find significant results. 


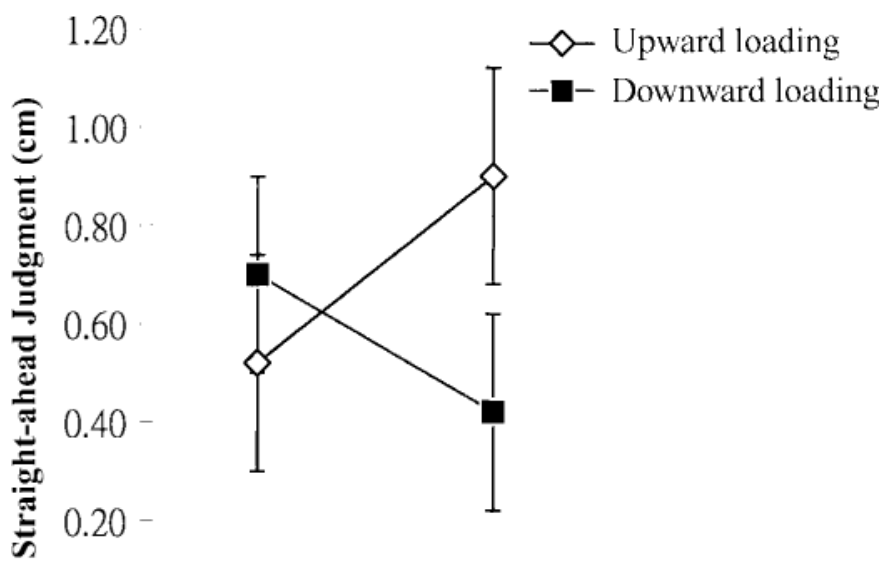

\section{$0.00 \frac{1}{\text { Before loading After loading }}$}

Figure 4. Mean interaction (averaged across 10 subjects) between the perceived horizontal judgments made before and after loading (error bars represent standard error).

To compare the variability of the present judgments with those of Grover and Craske's (1991) study, coefficients of variation $(\mathrm{CoV}$; standard deviation divided by the mean) were calculated (see Table 2). This comparison is valid since both studies used 10 subjects, 10 trials for each condition, and similar experimental designs. Table 2 shows that CoV values for the present study were considerably lower than those for the no-anesthetic conditions of Grover and Craske's study. This finding suggests that tongue position judgments along the vertical plane are more consistent than tongue position judgments along the horizontal plane.

\section{DISCUSSION}

The results of the present tongue-loading experiment are consistent with those of Grover and Craske's (1991) study. Similar outcomes were obtained from experiments on the loading of the limbs (McCloskey, 1978) and of the eyes (Skavenski, Haddad, \& Steinman, 1972). These findings suggest that the understanding of the mechanisms involved in the aftereffects observed in the limbs and in the eyes might be useful for understanding the mechanisms that govern the position sense of the tongue.

The eye has no joint and has few kinesthetic receptors; by contrast, the limb's position sense is conveyed by receptors in joints and tendons, and by muscle spindles (McCloskey, 1978). The effect of loading on the eyes was found to be much larger than that of the limb. For example, errors of as much as $90^{\circ}$ were found in the judgments of eye position after loading (Skavenski et al., 1972), whereas a bias of $4.3^{\circ}$ in loaded limbs was reported by McCloskey (1973). In spite of the difference in the magnitude of the aftereffect produced by loading, the

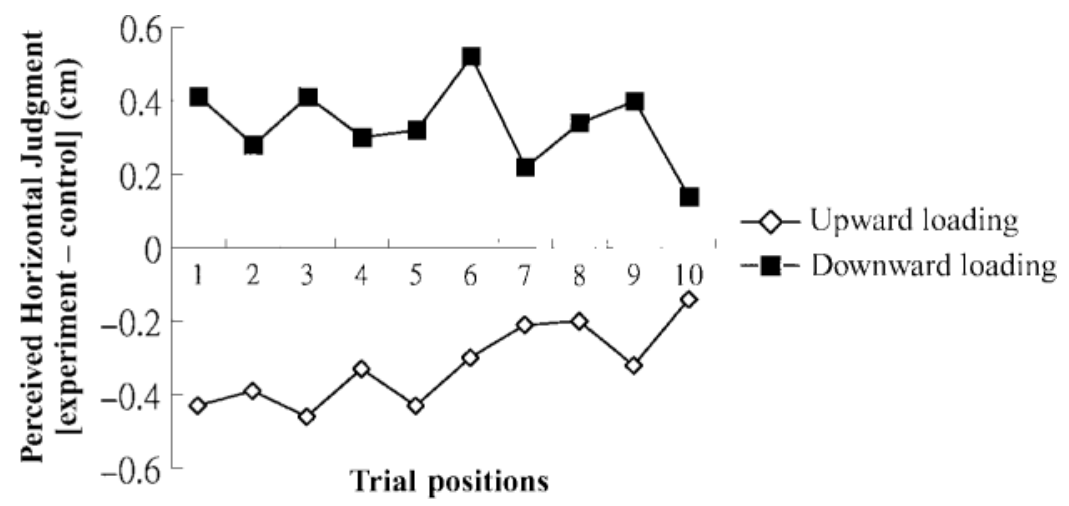

Figure 5. Graph showing the serial position of trials and the effect of tongue loading. 
Table 2

Comparison of Coefficients of Variation $(\mathrm{CoV})$ of the Present Study and Those of Grover and Craske's (1991) Study Across Different Experimental Conditions

\begin{tabular}{lccc}
\hline & \multicolumn{3}{c}{ CoV } \\
\cline { 2 - 4 } Tongue & Basic & Protrusion \\
Coading & Control & Control & $\begin{array}{c}\text { After } \\
\text { Loading }\end{array}$ \\
\hline \multirow{4}{*}{ Upresent } & Experiment \\
Downward & 0.59 & 0.78 & 0.88 \\
& 0.86 & 1.00 & 0.60 \\
Right & Grover $\&$ & Craske & \\
Left & 2.70 & 3.09 & 7.77 \\
\hline
\end{tabular}

direction of the bias of the position sense was identical. That is, after loading, both the limbs and the eyes are perceived to occupy a position farther in the direction opposite to the direction of the effort than is correct after loading (Craske, Crawshaw, \& Heron, 1975; McCloskey, 1973). Researchers have speculated that the observed bias might be due to the persistence of muscular tension or "postcontraction discharge" of the limb muscles after loading (Hutton, Smith, \& Eldred, 1973). Under normal circumstances, the brain requires correct information about the muscle length and the state of the tendon to accurately achieve a voluntary movement. Due to the postcontraction discharge, the limb would move in the direction of the previous effort. Consequently, when a subject is asked to place the limb at a certain position after loading, the judgment will be biased toward the direction of the previous effort. In their study of the postcontraction effect on the limb position sense in humans, Gregory, Morgan, \& Proske (1988) suggested that the bias of the limb position could be viewed as part of the postcontraction effect.

Anatomically, the body of the tongue consists almost entirely of interwoven muscles. The intrinsic muscles all have points of attachment within the body of the tongue. They run longitudinally, transversely, and vertically within the tongue. In general, the intrinsic tongue muscles alter the shape of the tongue by narrowing and flattening the blade, and lifting and lowering the tongue tip. The extrinsic muscles of the tongue have several points of attachment external to the tongue. These attachment points include the styloid process, the hyoid bone, the soft palate, and the jaw. The extrinsic muscles are involved in positioning the tongue body inside the oral and pharyngeal cavities. They also move the tongue outside the oral cavity, as in protrusion and lateralization (Zemlin, 1981). Given that the bias in the judgment of tongue position illustrated in the present study has the same direction as that observed under similar circumstances in the limb and the eye, it seems reasonable to suggest that the same mechanism is affecting the lingual position judgment after loading. Therefore, the spindles in the muscles of the tongue may continue to supply afferent information to the brain after the load is removed. If the afferent in- formation continues to signal a state of contraction after the load has been removed, an inaccurate estimation of current position will occur. It is also likely that the corollary discharge from the brain, which is associated with voluntary movements, is one of the contributors to the shift in perceived position of the tongue.

The main protruders in the tongue-loading experiments are the posterior fibers of the genioglossus. It is likely that the elevators, such as the styloglossus (SG) and palatoglossus (PG), work together with the longitudinalis superior (SL) to strain against downward loading, whereas the hyoglossus (HG) together with the longitudinalis inferior (IL) work against upward loading (see Hardcastle, 1976). Since the contractions of SG, PG, and HG primarily affect the height of the dorsum rather than the tongue tip and blade, SL and IL are believed to be the active muscles in opposing downward and upward loading, respectively, in the present experiment. The postcontraction discharge of the muscle spindles in the strained muscles caused by tongue loading could produce a movement of the tongue in the direction of the previous effort after the removal of the load. Although the spindle on the flaccid part of the tongue may fire to signal stretch, there is no relevant corollary discharge for recalibration, because the postcontraction discharge induced by the loading is not volitional. When subjects were asked to make judgments of vertical positions using the adjustment method, the corollary discharge associated with this intended movement continued to underrepresent the extent of actual movement in the direction opposite that of the previous effort. Consequently, the registered vertical position of the tongue was shifted in the direction of the previous effort.

There is general agreement that speech motor control requires the integration of multiple sensory signals with internally specified general motor goals (van der Merwe, 1997) and that target-reaching movements of the limbs are highly adaptive to disturbances (Barlow, 1998). To reach for a visual target, one has to transform information of the target position presented in the visual domain to muscular force exerted to the skeletal system for the reaching action. One way of conceptualizing the adaptive nature of target-reaching movement is that the image of the target and the proprioceptive coding of the initial position of the limb are transformed into common noetic coordinates before the CNS specifies a trajectory of the target-reaching movement (Redding \& Wallace, 2002). Under normal circumstances, (1) the felt limb position signaled by proprioceptors (muscle spindles, GTOs), joint receptors, and mechanoreceptors located within skin and muscles and (2) the seen limb position are identical. In order to investigate the mechanism of the adaptation, researchers have created discrepancies between the felt and seen limb positions by using virtual environment systems (Groen \& Werkhoven, 1998) or prism (Redding \& Wallace, 1990, 1992) that displace the entire visual field to one side. The typical finding from such 
studies is that the visual sense dominates the other senses in target-reaching movements; the visually displaced hand is actually felt where it is seen, a phenomenon termed immediate visual capture (Welch \& Warren, 1980) or strategic calibration (Redding \& Wallace, 2001, 2002). Experiments in which subjects wear prisms for longer periods of about 5 to $10 \mathrm{~min}$ show that the eye-hand coordination will make an adaptive change to deal with the new relationship between the seen and the felt limb position (Bedford, 1999). This adaptation results in the subject's reaching to the opposite side of the prism displacement for a short period of time after the prisms are removed (the aftereffect) (Bedford, 1993, 1999). Redding and Wallace $(1996,1997)$ theorize that prismatic adaptation is a remapping between the internal maps of visual space and the proprioceptive "limb space." When the relationship between the visual space and the limb space is changed, the brain is capable of making an adjustment of the spatial mapping function that links both spaces (Bedford, 1989). This explains the short-term negative aftereffects when the device causing the displacement is removed. Recent neurobiological evidence on monkeys and humans (Graziano \& Gross, 1998) shows that neurons in the brain encode the locations of visual, tactile, auditory, and remembered stimuli with respect to different parts of the body. This further supports the theory that different movable body parts have their own underlying maps, and that recoding is limited to a region surrounding the part of space where the disturbance is experienced (Bizzi \& Mussa-Ivaldi, 1998).

Similar to other movable parts of the body, the oral system is highly coordinated, flexible, and adaptive (Barlow, 1998). Although the literature on spatial mapping does not refer specifically to the tongue, both the present study and Grover and Craske's (1991) study replicated the aftereffect of tongue loading and suggest that the tongue has a position sense and is likely to be associated with an underlying spatial map. In the present study, subjects were asked to reach for the perceived horizontal target with their tongues after a period of loading, which is similar to the aftereffect of the prismatic adaptation. The horizontal target in the present experiment can be made available by the oral-lingual system and the vestibular system as a result of repeated exposure to sensory signals coming from the moving parts when they interact with the environment (Bizzi \& Mussa-Ivaldi, 1998). The tongue position can be given by transformed proprioceptive input, since visual information is not available. The tongue-loading procedure distorted the proprioceptive input and thus the internal map of the tongue position. As a result, a mapping error might have occurred, giving rise to the aftereffect demonstrated in the experiment. The higher variability found in the judgment along the horizontal axis (Grover \& Craske, 1991), relative to the variability found in the present study, may suggest that the underlying mapping of the coordinates along the vertical axis has a much finer resolution than the mapping of the coordinate along the horizontal axis.

\section{REFERENCES}

Abbs, J. H., \& Gracco, V. L. (1984). Control of complex motor gestures: Orofacial muscle responses to load perturbations of the lip during speech. Journal of Neurophysiology, 51, 705-723.

BARLOW, S. M. (1998). Real time modulation of speech-orofacial motor performance by means of motion sense. Journal of Communication Disorders, 31, 511-534.

BEDFORD, F. L. (1989). Constraints on learning new mappings between perceptual dimensions. Journal of Experimental Psychology: Human Perception \& Performance, 15, 232-248.

BEDFORD, F. L. (1993). Perceptual and cognitive spatial learning. Journal of Experimental Psychology: Human Perception \& Performance, 19, 517-530.

BEDFORD, F. L. (1999). Keeping perception accurate. Trends in Cognitive Sciences, 3, 4-11.

BizZI, E., \& Mussa-Ivaldi, F. A. (1998). Neural basis of motor control and its cognitive implications. Trends in Cognitive Sciences, 2, 97-102.

Borden, G. J., Harris, K. S., \& RaPhaEL, L. J. (1994). Speech science primer: Physiology, acoustics, and perception of speech (3rd ed.). Baltimore: Lippincott, Williams \& Wilkins.

BroekhuiJsen, M. L., \& van Willigen, J. D. (1983). Factors influencing jaw position sense in man. Archives of Oral Biology, 28, 387-391.

BrooKs, V. B. (1986). The neural basis of motor control. New York: Oxford University Press.

Craske, B., Crawshaw, M., \& Heron, P. (1975). Disturbance of the oculomotor system due to lateral fixation. Quarterly Journal of Experimental Psychology, 27, 459-465.

Darley, F. L., Aronson, A. E., \& Brown, J. R. (1975). Motor speech disorders. Philadelphia: W. B. Saunders.

EvarTs, E. V. (1981). Role of motor cortex in voluntary movements in primates. In V. B. Brooks (Ed.), Handbook of physiology: Section 1. The nervous system: Vol. 2. Motor control (pp. 1083-1120). Bethesda, MD: American Physiological Society.

FucCI, D. J., \& Lass, N. J. (1999). Fundamentals of speech science. Boston: Allyn \& Bacon.

Gracco, V. L. (1995). Central and peripheral components in the control of speech movements. In F. Bell-Berti \& L. J. Raphael (Eds.), Producing speech: Contemporary issues, for Katherine Safford Harris. New York: AIP.

Gracco, V. L., \& ABBs, J. H. (1985). Dynamic control of the perioral system during speech: Kinematic analyses of autogenic and nonautogenic sensorimotor processes. Journal of Neurophysiology, 54, 418-432.

Gracco, V. L., \& AbBs, J. H. (1988). Central patterning of speech movements. Experimental Brain Research, 71, 515-526.

GraZiano, M. S. A., \& Gross, C. G. (1998). Spatial maps for the control of movement. Current Opinion in Neurobiology, 8, 195-201.

Gregory, J. E., Morgan, D. L., \& Proske, U. (19 $\overline{8} 8)$. Aftereffects in the responses of cat muscle spindles and errors of limb position sense in man. Journal of Neurophysiology, 59, 1220-1230.

Groen, J., \& WerkHOVEN, P. J. (1998). Visuomotor adaptation to virtual hand position in interactive virtual environments. Presence, 7, 429-446.

Grover, C., \& Craske, B. (1991). The effect of loading on position sense in the tongue. Perception \& Psychophysics, 50, 7-14.

Hardcastle, W. J. (1976). Physiology of speech production. London: Academic Press.

Hayden, D. A., \& Square, P. A. (1994). Motor speech treatment hierarchy: A systems approach. Clinical Communication Disorders, 4 , 162-174.

Hutton, R., Smith, J., \& Eldred, E. (1973). Postcontraction sensory discharge from muscle and its source. Journal of Neurophysiology, 36, 1090-1103.

Kelso, J. A., Tuller, B., Vatikiotis-Bateson, E., \& Fowler, C. A. (1984). Functionally specific articulatory cooperation following jaw perturbations during speech: Evidence for coordinative structures. Journal of Experimental Psychology: Human Perception \& Performance, 10, 812-832.

LADEFOGED, P. (1975). A course in phonetics. New York: Harcourt Brace Jovanovich.

LADEFOGED, P., \& MADDIESON, I. (1996). The sounds of the world's languages. Cambridge, MA: Blackwell. 
Lowe, A. A. (1981). The neural regulation of tongue movements. Progress in Neurobiology, 15, 295-344.

MacNeilage, P. F. (1970). Motor control of serial ordering of speech. Psychological Review, 77, 182-196.

MCCLOSKEY, D. I. (1973). Differences between the senses of movement and position shown by the effects of loading and vibration of muscles in man. Brain Research, 61, 119-131.

McCloskEY, D. I. (1978). Kinesthetic sensibility. Physiological Reviews, 58, 763-820.

PERKELL, J. (1981). On the use of feedback in speech production. In T. Myers, J. Laver, \& J. Anderson (Eds.), The cognitive representation of speech (pp. 45-57). Amsterdam: North-Holland.

RedDing, G. M., \& Wallace, B. (1990). Effects on prism adaptation of duration and a timing of visual feedback during pointing. Journal of Motor Behavior, 22, 209-224.

REDDING, G. M., \& WALLACE, B. (1992). Effects on pointing rate and availability of visual feedback on visual and proprioceptive components of prism adaptation. Journal of Motor Behavior, 24, 226-237.

Redding, G. M., \& Wallace, B. (1996). Adaptive spatial alignment and strategic perceptual-motor control. Journal of Experimental Psychology: Human Perception \& Performance, 22, 379-394.

ReDDing, G. M., \& Wallace, B. (1997). Prism adaptation during target pointing from visible and nonvisible starting locations. Journal of Motor Behavior, 29, 119-130.
Redding, G. M., \& Wallace, B. (2001). Calibration and alignment are separable: Evidence from prism adaptation. Journal of Motor Behavior, 33, 401-412.

REDDING, G. M., \& WALlace, B. (2002). Strategic calibration and spatial alignment: A model from prism adaptation. Journal of Motor Behavior, 34, 126-138.

SkAVEnsKI, A. A., Haddad, G., \& Steinman, R. M. (1972). The extraretinal signal for the visual perception of direction. Perception \& Psychophysics, 11, 287-290.

VAN DER MERWE, A. (1997). A theoretical framework for the characterization of pathological speech sensorimotor control. In M. R. McNeil (Ed.), Clinical management of sensorimotor speech disorders (pp. 1-25). New York: Thieme.

Welch, R. B., \& Warren, D. H. (1980). Immediate perceptual response to intersensory discrepancy. Psychological Bulletin, 88, 638667.

ZEMLIN, W. R. (1981). Speech and hearing sciences: Anatomy and physiology (2nd ed.). Englewood Cliffs, NJ: Prentice-Hall.

(Manuscript received November 6, 2002; revision accepted for publication December 19, 2003.) 